\title{
Postsynaptic density protein 95 (PSD-95) is transported by KIF5 to dendritic regions
}

\author{
Ki-Seo Yoo ${ }^{1}$, Kina Lee ${ }^{1}$, Jun-Young Oh ${ }^{1,2}$, Hyoeun Lee ${ }^{2}$, Hyungju Park², Young Seok Park ${ }^{3}$ and Hyong Kyu Kim ${ }^{1 *}$ (D)
}

\begin{abstract}
Postsynaptic density protein 95 (PSD-95) is a pivotal postsynaptic scaffolding protein in excitatory neurons. Although the transport and regulation of PSD-95 in synaptic regions is well understood, dendritic transport of PSD95 before synaptic localization still remains to be clarified. To evaluate the role of KIF5, conventional kinesin, in the dendritic transport of PSD-95 protein, we expressed a transport defective form of KIF5A $(\Delta M D)$ that does not contain the $\mathrm{N}$-terminal motor domain. Expression of $\triangle M D$ significantly decreased PSD-95 level in the dendrites. Consistently, KIF5 was associated with PSD-95 in in vitro and in vivo assays. This interaction was mediated by the Cterminal tail regions of KIF5A and the third PDZ domain of PSD-95. Additionally, the ADPDZ3 (the association domain of NMDA receptor and PDZ3 domain) expression significantly reduced the levels of PSD-95, glutamate receptor 1 (GluA1) in dendrites. The association between PSD-95 and KIF5A was dose-dependent on Staufen protein, suggesting that the Staufen plays a role as a regulatory role in the association. Taken together, our data suggest a new mechanism for dendritic transport of the AMPA receptor-PSD-95.
\end{abstract}

Keywords: PSD-95, KIF5, Glutamate receptor 1, Dendritic transport

\section{Introduction}

In excitatory neurons, neuronal excitability changes synaptic function [1-5] by regulating expression level of synaptic scaffold proteins such as postsynaptic density protein 95 (PSD-95) - a member of the membraneassociated guanylate kinase (MAGUK) class of proteins at synapses [6-8]. PSD-95 regulates the trafficking and localization of glutamate receptors such as $\alpha$-amino-3-hydroxy-5-methyl-4-isoxazolepropionic acid (AMPA)-type or $N$-methyl-D-aspartate (NMDA) type-receptors [4]. Overexpression of PSD-95 enhances the amplitude of the AMPA receptor-mediated synaptic current $[1,9,10]$. For all these reasons, PSD-95 has been implicated in synaptic development, plasticity [11, 12], and defects across several disorders $[13,14]$. Despite essential roles of PSD-95 in synaptic functions, its transport and localization to dendrites are partially understood [15]. One study reported that kinesin superfamily protein $1 \mathrm{~B} \alpha(\mathrm{KIF} 1 \mathrm{~B} \alpha)$ - a member of the kinesin-3 family-associates with MAGUKs such as PSD-95 and synapse-associated protein (SAP)-97,

\footnotetext{
* Correspondence: hkkim69@chungbuk.ac.kr

${ }^{1}$ Department of Medicine and Microbiology, Graduate Program in

Neuroscience, College of Medicine, Chungbuk National University, 1

Chungdae-ro, Seowon-gu, Cheongju 28644, South Korea

Full list of author information is available at the end of the article
}

as well as synaptic scaffolding molecule (S-SCAM) and membrane associated guanylate kinase inverted-2 (MAGI2 ), suggesting that KIF1B $\alpha$ functions as a motor protein for synaptic localization of these proteins [15].

KIF proteins transport a various molecules, including proteins, synaptic vesicles, and mitochondria along the microtubules cytoskeleton of an axon or dendrites to synaptic regions [16]. In particular, KIF5, which belongs to the recently classified kinesin-1 family, consists of three isoforms (A, B and C) [17]. It transports ribonucleoprotein complexes, synaptic vesicles, mitochondria, AMPA receptor vesicles, tyrosine receptor kinase B (TrkB)-containing vesicles [18], and $\gamma$-aminobutyric acid $\left(\mathrm{GABA}_{\mathrm{A}}\right)$ receptor vesicles [19] in neurons, as well as slowly transported cargo proteins in axons [20]. KIF5 localizes AMPA receptor vesicles to the postsynaptic regions interacting with glutamate receptor interacting protein 1 (GRIP1) - a scaffolding protein similar to a MAGUK and a member of the PSD-95/SAP90/discs large homology (DLG)/zona occludens (ZO)-1 (PDZ)domain proteins, functioning as a synaptic scaffolding protein [21, 22]. Several studies have reported that MAGUKs are transported to membrane regions by KIF13B [23, 24].

C The Author(s). 2019 Open Access This article is distributed under the terms of the Creative Commons Attribution 4.0 International License (http://creativecommons.org/licenses/by/4.0/), which permits unrestricted use, distribution, and 
In the present study, we revealed that KIF5 as a motor protein involved in PSD-95 dendritic transport. The Cterminal tail region of KIF5A was associated with the PDZ3 domain of PSD-95. The expression of the ADPDZ3 domain, which includes an NMDA receptorassociated domain (AD), significantly decreased levels of PSD-95 and surface glutamate receptor 1 (GluA1) at the postsynaptic site. Finally, we found that the KIF5A-PSD95 complex colocalized with GluA1-immuopositive particles in dendritic regions, indicating that KIF5A mediates the transport of both PSD-95 and GluA1containing vesicles. Thus, we suggest that PSD-95 works as both a scaffolding protein in the excitatory synapses and an adaptor between a cargo and motor proteins.

\section{Results}

Expression of a dominant-negative form of KIF5A reduces level of PSD-95 in dendrites

To examine the relevance of kinesin motor protein to dendritic transport of PSD-95, we expressed either green fluorescent protein (GFP), GFP-tagged wild type (WT) KIF5A (which is enriched in neurons) [17], or a dominant-negative mutant of KIF5A lacking the N-terminal motor domain $(\triangle \mathrm{MD})[25]$ in cultured hippocampal neurons. We then examined PSD-95 particles in dendrites. Consistent with our previous results [26], $\triangle \mathrm{MD}$ expression significantly reduced the number and average size of PSD-95 particles by 78.48 and $61.71 \%$ (Fig. 1), respectively, indicating that inhibition of KIF5A functions reduces PSD-95 levels in dendrites. Interestingly, expression of the WT did not induce any significant change.

\section{PSD-95 colocalizes with KIF5}

In order to specify which isoform of KIF5 interacts with the PSD-95, we examined an association between isoform of KIF5 and PSD-95. The results showed that the PSD-95 interacts with all isoforms of KIF5s (Additional file 1: Figure S1). We examined the localization of PSD-95 and KIF5 in cultured neurons. As shown in Fig. 2a and b, a significant number of puncta immunopositive for KIF5 were colocalized with puncta of PSD-95 (PSD-95/KIF5: $53.17 \% \pm 3.86 \%)$ and many puncta immunopositive for PSD-95 were colocalized with puncta of KIF5 (KIF5/PSD95: $62.55 \% \pm 1.69 \%)$. To further visualize the colocalization of two proteins in vivo, we performed a proximity ligation assay (PLA), which also indicated an association between the two proteins. The results of PLA showed that endogenous KIF5 and PSD-95 interacts in dendrites (Fig. 2c). Supporting this result, our immunoprecipitation analysis using anti-KIF5 or anti-PSD-95 antibody and rat brain lysates also showed interaction between endogenous KIF5 and PSD-95 (Fig. 2d). Taken together, these results show that PSD-95 may interact with KIF5 in neurons. Although all KIF5 members had showed the interaction with
PSD-95 (Additional file 1: Figure S1), we decided to focus on the role of KIF5A, having pan-neuronal expression pattern [17], to study detailed mechanisms of the interaction and dendritic transport.

Interaction between PSD-95 and KIF5A requires the PDZ3 domain of PSD-95

In order to identify which domains of PSD-95 and KIF5A are required for this interaction, we constructed a series of truncated mutants of PSD-95 and KIF5A. First, we examined whether FLAGtagged full-length KIF5A could interact with hemagglutinin (HA)-tagged truncated PSD-95 mutants. As shown in Fig. 3a and c, the third PDZ domain (PDZ3) was required for interaction with KIF5A. Next, we examined whether HA-tagged fulllength PSD-95 could interact with FLAG-tagged truncated KIF5A mutants. Consistent with previous reports $[27,28]$, the results indicated that the tail region of KIF5A was required for the interaction (Fig. 3d). To identify the necessary regions more closely, we constructed plasmids to express either the PDZassociated domain of NMDA receptors (AD) or PDZ3 and examined the interaction with full-length KIF5A. The PDZ3 domain was sufficient for the interaction with KIF5A, although this interaction was weaker than that with ADPDZ3 (Fig. 4). Thus, in the following experiments, we used ADPDZ3 to block the interaction between the two proteins. Our data indicate that the tail region of KIF5A and PDZ3 domain of PSD-95 are required for the interaction between KIF5A and PSD-95.

\section{ADPDZ3 expression reduces PSD-95 level in dendrites}

Because ADPDZ3 was required for the interaction between KIF5A and PSD-95 (Fig. 4), we examined whether ADPDZ3 expression affects the dendritic level of PSD95. The cultured hippocampal neurons were infected with Sindbis viruses encoding either GFP or GFPADPDZ3 and subjected to immunostaining using monoclonal anti-PSD-95 antibody or polyclonal anti-synapsin I antibody. The monoclonal PSD-95 antibody did not detect overexpressed ADPDZ3 particles (data not shown). ADPDZ3 domain expression significantly reduced the number of PSD-95 particles, but not synapsin I particles (Fig. $5 \mathrm{a}-\mathrm{c}$ ), suggesting that inhibition of the interaction between PSD-95 and KIF5A blocks PSD-95 dendritic localization. This reduction was more dramatic in distal dendritic regions than in proximal regions (Additional file 2: Figure S2). Accordingly, ADPDZ3 expression resulted in a significant reduction of synapse number, possibly due to the reduced dendritic transport of PSD-95 (Fig. 5a, d). 

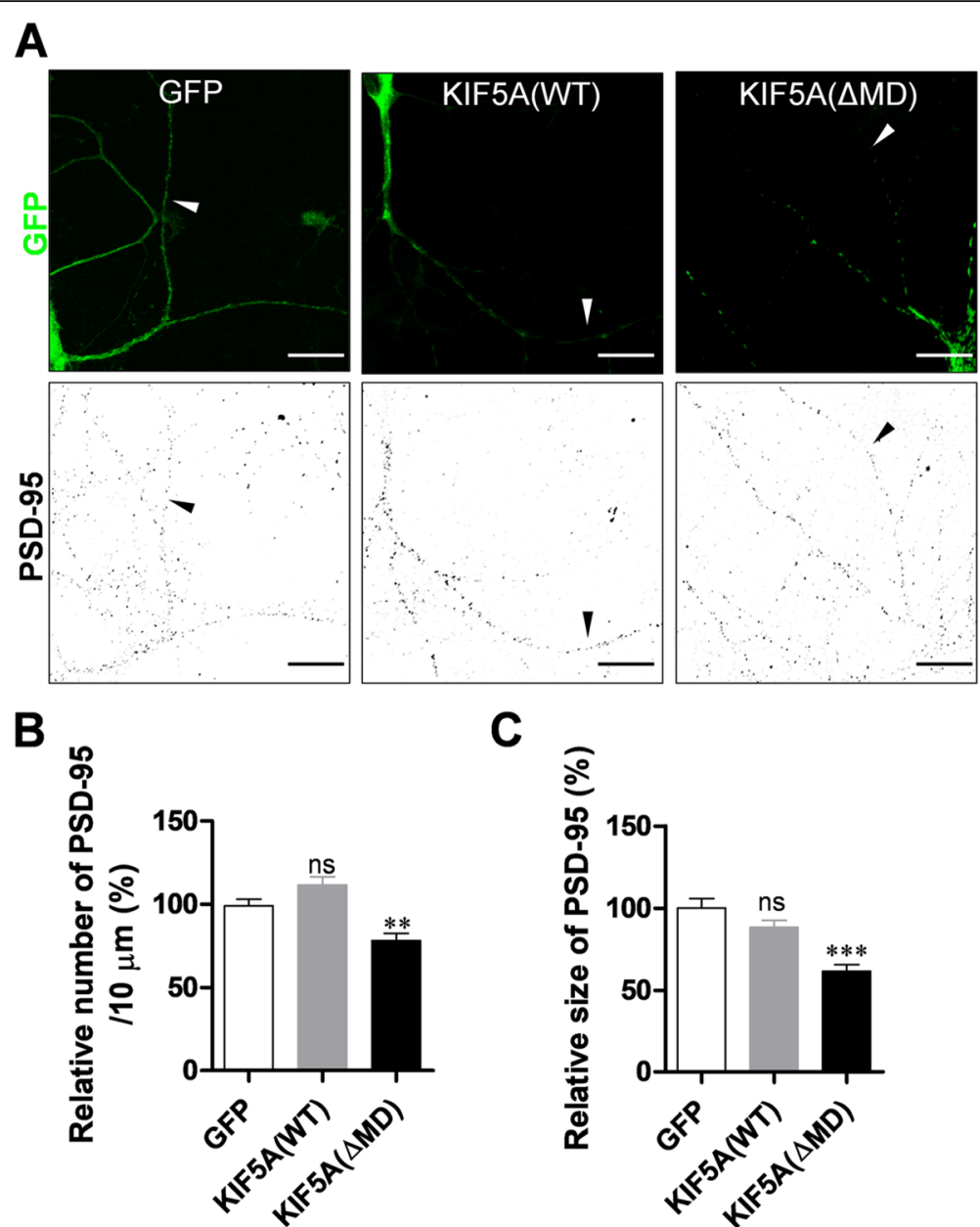

Fig. 1 Expression of KIF5A $\triangle M D$ mutant reduces the number and average size of PSD-95 particles in dendrites. Cultured rat hippocampal neurons were infected with Sindbis viruses encoding GFP, GFP-KIF5A (WT), or GFP-KIF5A ( $\Delta M D)$, incubated for $9 \mathrm{~h}$, and immunostained with monoclonal anti-PSD-95 antibody. a Representative images of infected neurons. Scale bar: $20 \mu \mathrm{m}$. b $\Delta \mathrm{MD}$ expression significantly reduces the number of PSD-95 particles (GFP: 100.00\% $\pm 3.88 \%, n=31$ dendrites, $3417 \mu$ m; KIF5A [WT]: $111.70 \% \pm 4.91 \%, n=31$ dendrites, $3724 \mu \mathrm{m}$; KIF5A $[\Delta M D]: 78.48 \% \pm 4.23 \%, n=29$ dendrites, $3287 \mu$ m; Kruskal-Wallis test: $P<0.0001$; Dunn's multiple comparison test: ${ }^{*} P<0.01$, ns: not significant). c $\triangle M D$ expression significantly reduces the average size of PSD-95 particles (GFP: $100.00 \% \pm 5.83 \%, n=31$ dendrites; KIF5A [WT]: $88.39 \% \pm 4.27 \%$, $\mathrm{n}=31$ dendrites; KIF5A [ $\Delta \mathrm{MD}]: 61.71 \% \pm 4.00 \%, \mathrm{n}=29$ dendrites; Kruskal-Wallis test: $P<0.0001$; Dunn's multiple comparison test: ${ }^{* *} P<0.001, \mathrm{~ns}:$ not significant). $N$ values denote the dendritic number from $n$ neurons

\section{ADPDZ3 expression reduces surface GluA1 level in dendrites}

Since expression of the ADPDZ3 domain reduced the level of PSD-95-a major scaffolding protein in the synapses of excitatory neurons $[1,2,4,29]$, it is possible that expression of AMPA receptor at postsynaptic membranes is also affected. To test this idea, we examined whether ADPDZ3 expression also reduces the level of surface AMPA receptors. After ADPDZ3 expression, surface GluA1 was immunostained and evaluated by imaging analysis. Consistent with the results of our PSD-95 assay, ADPDZ3 expression significantly reduced the number of surface GluA1 particles (Fig. 6), supporting the idea that expression of AMPA receptor at synaptic membranes is dependent on KIF5A-mediated transport of PSD-95 to synaptic regions.

\section{Staufen modulates the association between PSD-95 and KIF5A}

Previous studies demonstrated that AMPA receptor vesicles are transported to the dendrites by KIF5 through the interaction between glutamate receptor interacting protein 1 (GRIP1) and kinesin heavy chain [22, 28]. Thus, we investigated whether KIF5 - associated with PSD-95 interacts with AMPA receptor vesicles. GFP-tagged PSD-95 was expressed in the cultured hippocampal neurons and immunostained using anti-KIF5 and GluA1 antibodies to analyze colocalization among PSD-95, KIF5, and GluA1. 
A
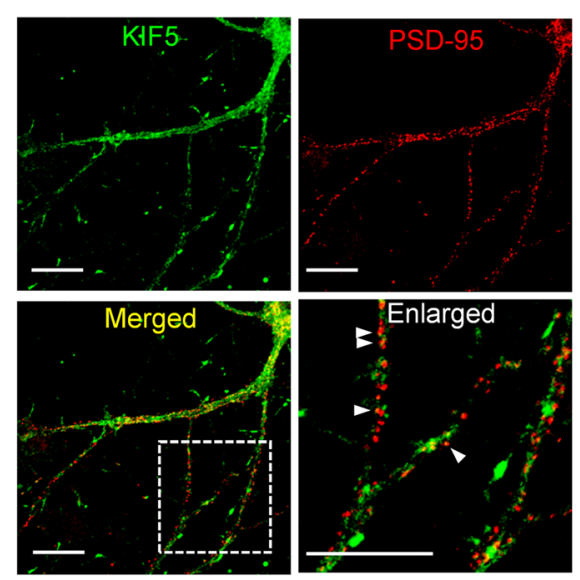

C
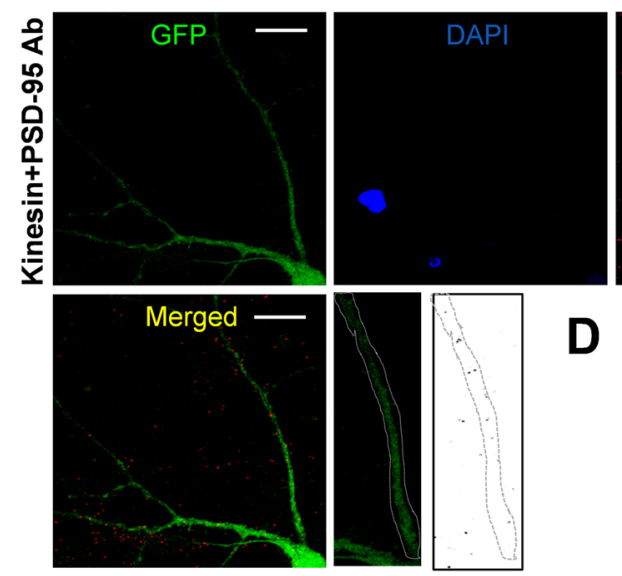

D

B
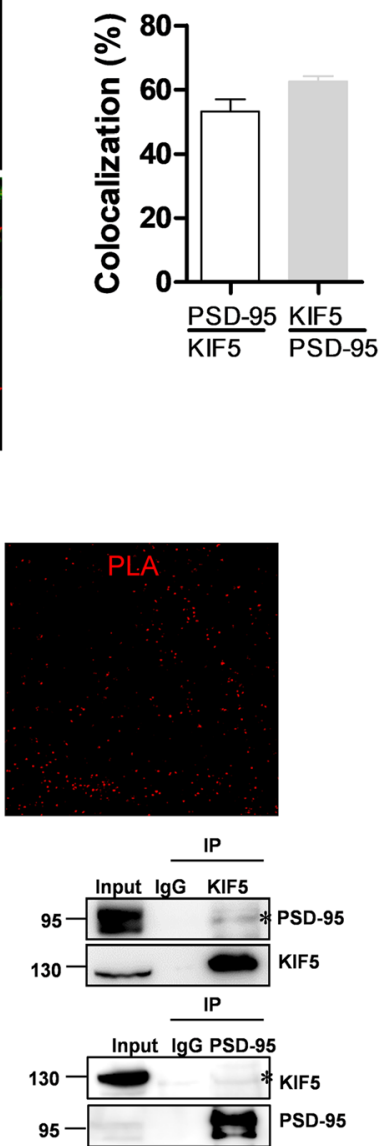

Fig. 2 PSD-95 is colocalized with KIF5. a Representative images of immunostaining. Scale bar: $20 \mu \mathrm{m}$. Cultured neurons were immunostained with polyclonal anti-PSD-95 antibody and monoclonal anti-KIF5 antibody (H2), followed by Alexa Fluor 488 anti-rabbit lgG antibody for PSD-95 and Cy3-conjugated anti-mouse lgG antibody for KIF5. b Results of co-localization analysis. 53.17\% $\pm 3.86 \%$ ( $n=19$ dendrites, $1354 \mu \mathrm{m})$ of KIF5immunopositve puncta are colocalized with PSD-95-immunopositve puncta and 62.55\% $\pm 1.69 \%$ ( $n=19$ dendrites, $1354 \mu$ m) of PSD-95immunopositive puncta are colocalized with KIF5-immunopositve puncta. c Results of proximity ligation assay. Cultured neurons were infected with Sindbis viruses encoding GFP and subjected to PLA. Red dots in PLA indicate an interaction between the two proteins. Scale bar: $20 \mu \mathrm{m}$. $\mathbf{d}$ Results of IP assays using rat brain lysates. In total $500 \mu \mathrm{g}$ of rat brain lysate was used in the IP assays using $3 \mu \mathrm{g}$ monoclonal anti-KIF5 antibodies or polyclonal PSD-95 antibodies. These were analyzed by Western blotting using the antibody indicated. Asterisks indicate interaction bands in the Western blot assays

As shown in Fig. 7a and b, we found colocalization among these three proteins in dendrites, suggesting that AMPA receptor vesicles are transported to the dendrites by PSD95 - KIF5A complexes. Next, we explored to identify whether any other linker or adaptor proteins are involved in the interaction between KIF5 and PSD-95. Because a previous study showed that Staufen functions as a linker protein for RNA granules, which are known as a cargo of KIF5 [30], and synaptic localization of PSD-95 depends on the expression or availability of Staufen [31], we examined whether Staufen expression modulates the association between PSD-95 with KIF5A. We gradually increased an amount of Myc-tagged Staufen to the cells expressing
FLAG-tagged KIF5A and HA-tagged PSD-95, and measured interactions between KIF5A and PSD-95 using immunoprecipitation. Our results showed that Staufen expression increased the interaction in dosedependent manner (Fig. 7c, d), indicating that Staufen functions as a linker or adaptor between cargoes and KIF5.

\section{Discussion}

In the present study, we provide evidence that KIF5 (kinesin-1 family), also known as conventional kinesin, is a motor protein for PSD-95 dendritic transport. A previous study [15] reported that KIF1B $\alpha$ (kinesin-3 family) 
A

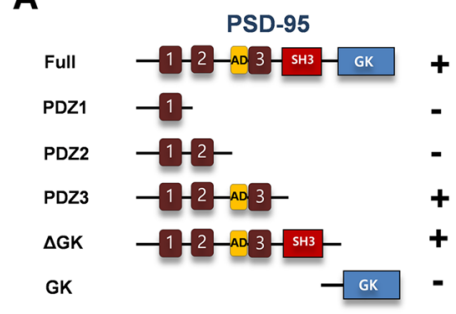

C

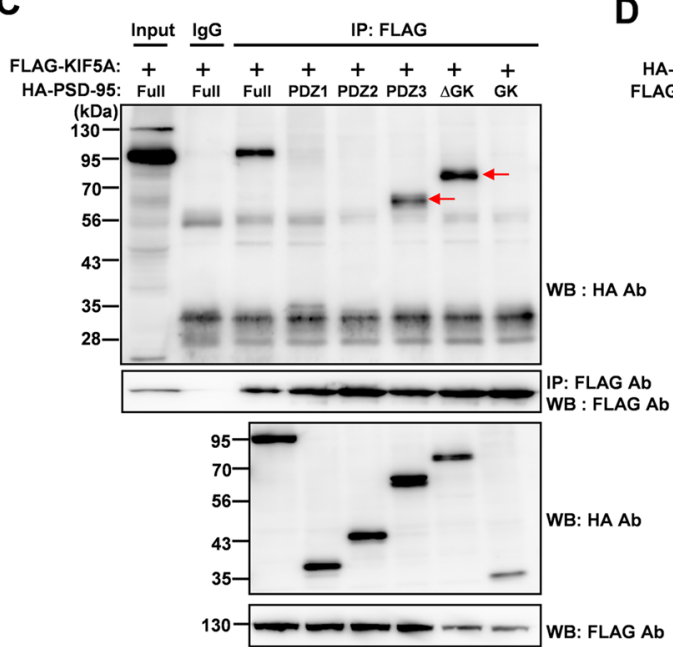

B

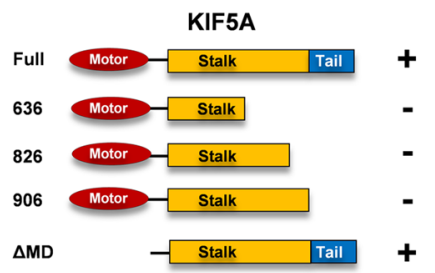

D

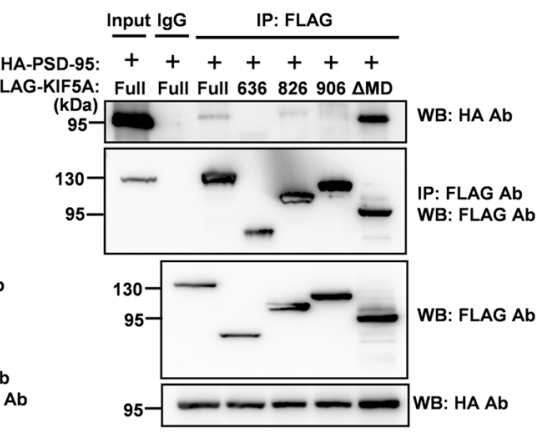

Fig. 3 The PDZ3 domain of PSD-95 and the tail region of KIF5A are required for the interaction. Full-length PSD-95 or truncated mutants with full-length KIF5A (a, c), and full-length KIF5A or truncated mutants with full-length PSD-95 (b, d) were transfected into HEK 293 T cells. The cell lysates were then used in immunoprecipitation assays. a Truncated mutants of PSD-95 and interaction with full-length KIF5A, analyzed by co-IP assays. GK: Guanosine monophosphate kinase. b Truncated mutants of KIF5A and interaction with full-length PSD-95, analyzed by co-IP assays. c, d Results of co-IP assays. The lysates were precipitated with anti-FLAG antibody, and the immunoprecipitates were analyzed in Western blotting assays using anti-HA antibody

associates with the C-terminal regions of PSD-95, while another [23] reported KIF13B (GAKIN) interacted with PSD-95 in epithelial cells. Lin et al. (2012) showed that KIF3A (kinesin-2 family) is involved in GluA2 trafficking in combination with GRIP 1 and PSD-95 in retina cells [32]. In the line with this idea, our results showed that inhibiting KIF5A by expressing a dominant-negative form ( $\triangle \mathrm{MD}$ ) did not completely block PSD-95 localization (Fig. 1). In addition to KIF5, we also identified interaction of PSD-95 with KIF3A (data not presented). These results indicate that multiple motor proteins are involved in PSD95 transport in various neuron types. Despite their somewhat overlapping expression level, each KIF displays a distinct expression pattern in the brain [33]. KIF5A is highly enriched in neurons of the cortex and the hippocampus [17, 25, 33], KIF1B in motor neurons in the medulla oblongata and the spinal cord [34], and KIF3A in granular cells of the cerebellum [35], while KIF13A in non-nerve tissues $[1,24]$. It is thus possible that dendritic transport of scaffolding proteins such as PSD95 is regulated by distinct KIF isoforms in different brain areas.
Many previous studies have indicated that PSD-95 work as an adaptor between a motor protein and receptor-containing vesicle cargoes. For example, Mint1/X11 (mLin-10) - a PDZ domain-containing protein-interlinks NMDA receptor-containing vesicles and KIF17, which is a member of kinesin-2 family [21]. Glutamate [NMDA] receptor subunit 1 (GluN1) and GluN2 - containing vesicles are transported to dendrites with SAP97 and calcium/calmodulin-dependent serine protein kinase (CASK), while PDZ domain-containing MAGUKs are transported by KIF17 [36]. In addition, by directly interacting with GRIP1, which is another PDZ domain-containing scaffolding protein, KIF5C is reported to transport GluA2 - containing vesicles to dendrites [28]. Thus, GRIP1 interlinks $N$-cadherin and GluA2containing vesicles, transporting them into the dendrites [22]. Interestingly, Huntingtin-associated protein 1 (HAP 1) works as an adaptor molecule for the dendritic transport of $\mathrm{GABA}_{\mathrm{A}}$ receptor-containing vesicles in the inhibitory synapses [37]. Scaffolding and motor proteins carry out dendritic transport and synaptic localization of transmembrane proteins, such as receptors [38]. Our 
A
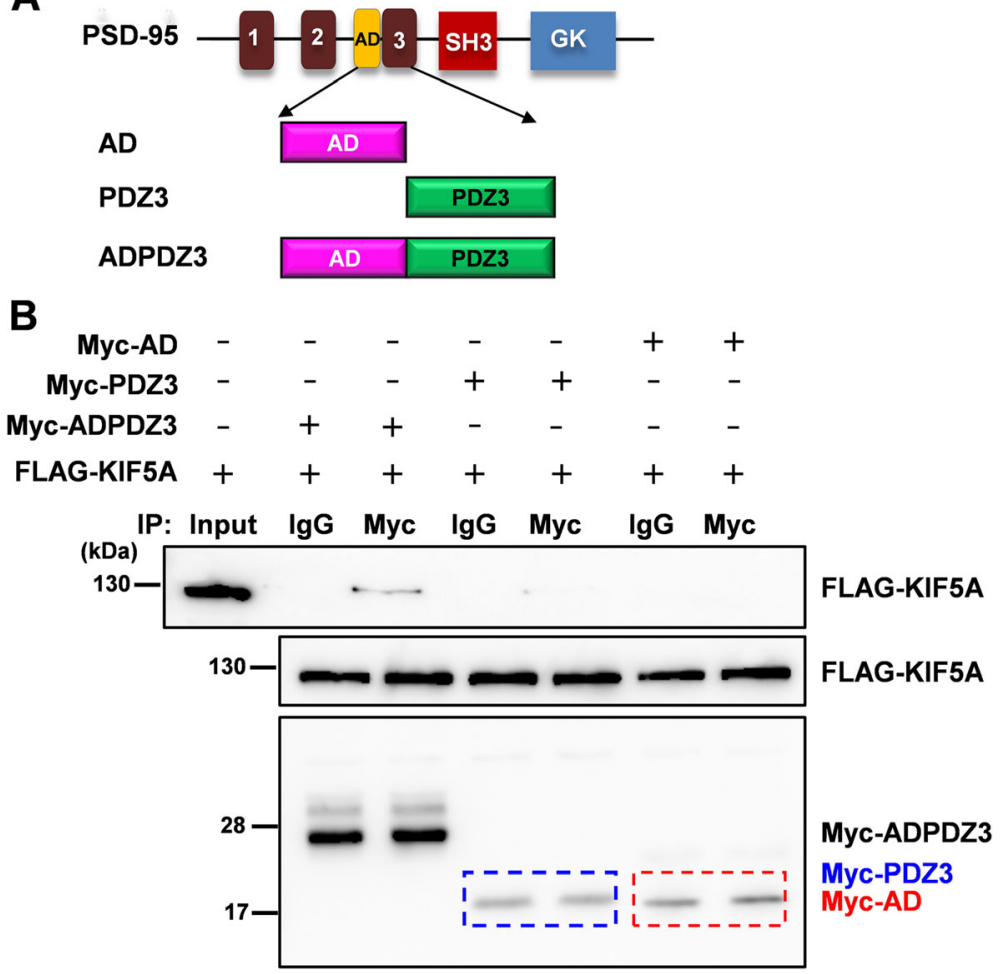

Fig. 4 ADPDZ3 domain is required for the interaction. To identify which domains are necessary for the interaction, AD (PDZ-associated domain of the NMDA receptor), PDZ3, ADPDZ3 were constructed and used in co-IP assays with full-length KIF5A. a Truncated mutants of PSD-95. b Results of co-IP assays. The blue dotted box indicates the expression of Myc-tagged PDZ3, while the red one indicate Myc-tagged AD

data agree with these previous results, because GluA1 expression on dendritic membranes was dependent on KIF5 - mediated transport of PSD-95 (Figs. 6, 7a).

GRIP 1 directly interacts with KIF5 through PDZ6 and PDZ7 domain, functioning as an adaptor between KIF5A and GluA2-containing vesicles [28]. The protein mLin-10 also directly interacts with KIF17 via the PDZ1 domain [21]. It is likely that PSD-95 directly interacts with KIF5A, although we did not examine this possibility in the study. However, a previous study identified a putative PDZ interaction motif (class I: Ser/Thr - X - Val, S/TXV) [39] in the tail regions of KIF5A, and this PDZ interaction motif was only found in the KIF5A isoform [17]. The present study corroborated previous data $[21,28]$, showing that PSD-95 binding to KIF5 may steer KIF5A to dendrites, as occurs when an adaptor binds to a motor protein. Another previous study suggested that PSD-95 is associated with Staufen in synaptic regions [31]. Staufen also works as an adaptor protein for KIF5 cargoes [30]. Consistent with these studies, in the present study, Staufen expression increased the association of PSD-95 with KIF5 (Fig. 7c and d), indicating that Staufen might modulate the PSD-95-KIF5 complex. Further studies should investigate the detailed molecular configuration of scaffolding protein-motor protein transport complexes modulated by Staufen.

Motor protein expression increases the levels of the corresponding cargo protein or associated protein in dendrites [22]. In the present experiment, even though the dominant mutant form of KIF5A ( $\triangle M D)$ significantly decreased the level of PSD-95 in dendrites, KIF5A (WT) did not increase PSD-95 transport (Fig. 1). Considering for multiple motors to be involved in the transport of PSD-95, the role of single protein might not be considerable. Alternatively, neuronal activity may be required to increase motor activity. Indeed, increases in expression levels of cargo proteins require neuronal activity [26]. The present study has suggested a new mechanism in the dendritic transport of PSD-95 and receptor-containing vesicles in glutamatergic synapses (Fig. 7e).

\section{Methods}

\section{Cell cultures and transfections}

Rat hippocampal neurons were isolated from 1-day-old pups (Sprague Dawely, Samtako, Osan, Republic of Korea). Cultures were maintained in Neurobasal-A (Life Technologies, Carlsbad, CA, USA) supplemented with B27 supplement (Life Technologies) at $37^{\circ} \mathrm{C}$ and in $5 \%$ 
A
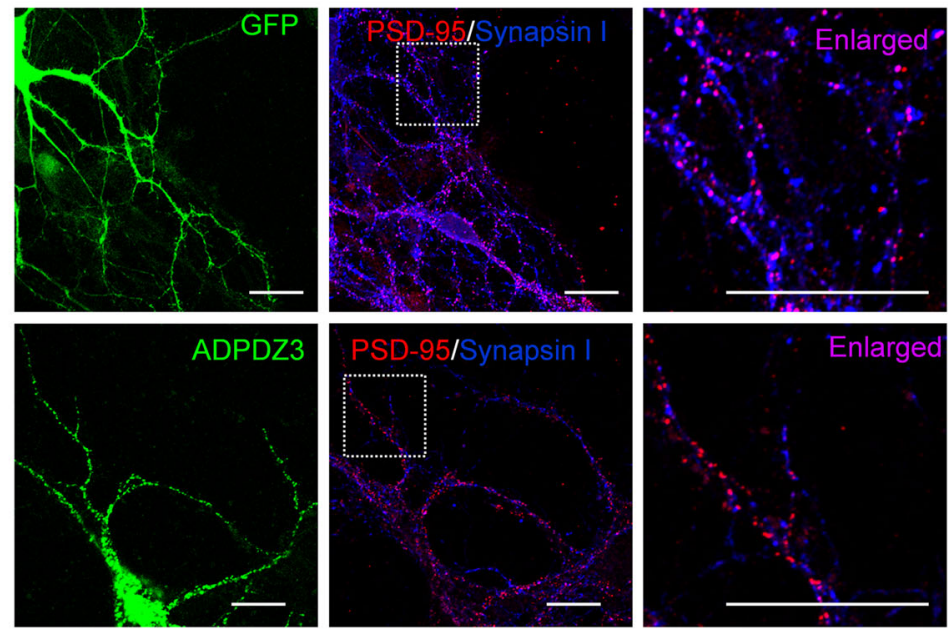

B
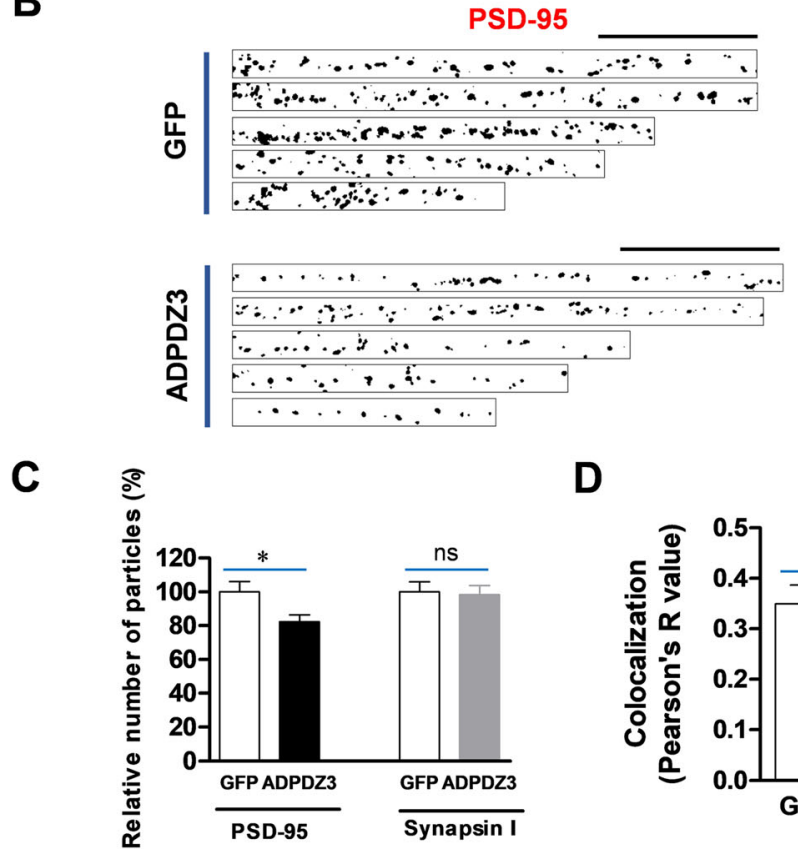

$\mathbf{D}$

Fig. 5 ADPDZ3 expression reduces the level of PSD-95 in dendrites. Cultured rat hippocampal neurons were infected with Sindbis viruses encoding GFP or GFP-ADPDZ3 and incubated for $9 \mathrm{~h}$ to allow expression. The cultures were then subjected to immunostaining using monoclonal anti-PSD-antibody and Cy3-conjugated anti-mouse lgG antibody or polyclonal anti-synapsin I antibody and Alexa Fluor 647 antirabbit IgG antibody. They were then visualized using confocal microscopy. a Representative images of expressed neurons. Arrows indicate analyzed dendrites. Scale bar: $20 \mu \mathrm{m}$. b, c ADPDZ3 expression reduced the number of PSD-95 particles in the dendrites (GFP: 100.00\% $\pm 6.08 \%$, $n=32,1766 \mu \mathrm{m}$; ADPDZ3: 82.27\% 4.03\%, $n=35,1973 \mu \mathrm{m}$; * $P<0.05$ ), but not that of synapsin I particles (GFP: $100.00 \% \pm 6.00 \%, n=32,1766 \mu \mathrm{m}$; ADPDZ3: $98.36 \% \pm 5.41 \%, n=35,1973 \mu \mathrm{m}$; ns: not significant). Scale bar: $20 \mu \mathrm{m}$. d ADPDZ3 expression significantly reduced colocalization between PSD-95 and synapsin I (GFP: $0.350 \pm 0.037, n=32$; ADPDZ3: $0.214 \pm 0.035, n=37 ;{ }^{* *} P<0.01$ ). N values indicate number of dendrites from multiple neurons derived from three different batches

$\mathrm{CO}_{2}$, as previously described [31]. The cultures were performed in accordance with the approved animal protocols and the guidelines of the Institutional Animal Care and Use Committee of Chungbuk National University (CBNUA-1049-17-01). Human embryonic kidney (HEK) $293 \mathrm{~T}$ cells were maintained in Dulbecco's Modified
Eagle's Medium (Life Technologies) supplemented with $10 \%$ fetal bovine serum (Biowest, Nuaillé, France). With regards to transfection of the neurons, target genes were subcloned to a pSinRep5 vector for Sindbis viral expressions and packaged into Sindbis virion particles according to Invitrogen's user manual (Invitrogen, Carlsbad, CA, 
A

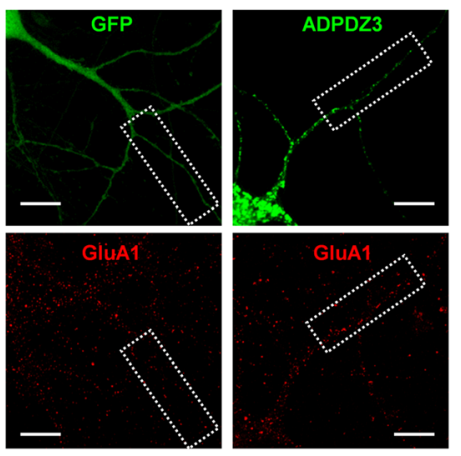

C

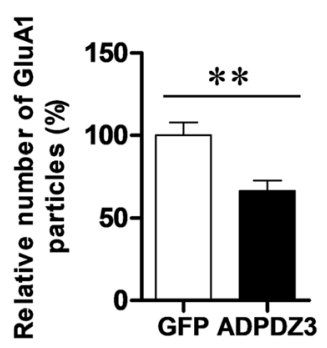

D

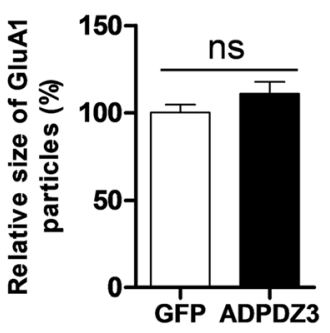

B

$$
\begin{aligned}
& \text { GFP } \\
& \text { ADPDZ3 }
\end{aligned}
$$

Fig. 6 ADPDZ3 expression reduces surface glutamate receptor 1 (GluA1) in dendrites. Cultured rat hippocampal neurons were infected with Sindbis viruses encoding GFP or GFP-ADPDZ3 and incubated for $9 \mathrm{~h}$ to allow expression. The cultures were then subjected to immunostaining using polyclonal GluA1 antibody and Cy3-conjugated anti-mouse IgG antibody. They were visualized using confocal microscopy. a, b Representative images of expressed neurons and selected dendrites in the analyses. Dotted boxes indicate analyzed dendrites. Scale bar: 20 um. c, d ADPDZ3 expression reduced the number of surface GluA1 particles (GFP: 100.00\% \pm 7.83\%, $n=18,1486 \mu \mathrm{m} ;$ ADPDZ3: $66.50 \% \pm 6.21 \%, n=14$, $1254 \mu \mathrm{m} ;{ }^{* *} P<0.01$ ), but not size of the surface GluA1 particles (GFP: $100.00 \% \pm 4.51 \%, n=18 ;$ ADPDZ3: $110.80 \% \pm 6.82 \%, n=14, n s: n o t$ significant). $\mathrm{N}$ values indicate number of dendrites from multiple neurons derived from three different batches

USA). The Sindibis virion was directly added to the cultured neurons and incubated for 6-12 h. Transfection of the HEK cells was carried out by transferring DNA constructs using a calcium phosphate method.

\section{DNA constructs}

Complete cDNA of mouse PSD-95 (disks large homolog 4, NM_007864) were amplified using PCR (mPSD95-R1$\mathrm{S} \rightarrow \mathrm{mPSD}$ 95-Xho-A) and inserted at the EcoRI/XhoI site of the pCS4-3xHA vector. To construct the mutant form of the PSD-95, full length or partial PSD-95 fragments were amplified using PCR and inserted at the EcoRI/XhoI site of the pCS4-3xHA vector (full length: mPSD95-R1$\mathrm{S} \rightarrow$ mPSD95-Xho-A; PDZ1: mPSD95-R1-S $\rightarrow$ PZD1-XhoA; PDZ1-2: mPSD95-R1-S $\rightarrow$ PZD2-Xho-A; PDZ1-3: mPSD95-R1-S $\rightarrow$ PZD3-Xho-A; $\triangle$ GK: mPSD95-R1$\mathrm{S} \rightarrow$ SH3-Xho-A; GK: GMPK-R1-S $\rightarrow$ mPSD95-Xho-A). To allow fine mapping, PSD-95 fragments were amplified using PCR (ADPDZ3: mPSD95-ADPDZ3-R1-S $\rightarrow$ PDZ3Xho-A2; PDZ3: mPSD95-PDZ3-R1-S $\rightarrow$ PDZ3-Xho-A2; AD: mPSD95-ADPDZ3-R1-S2 $\rightarrow$ AD-Xho-A). In the case of ADPD3 and PDZ3, the fragments were inserted at the EcoRI/XhoI site of the pCS4-3xHA vector. While in the case of $\mathrm{AD}$, they were inserted at the $E c o R \mathrm{I} / \mathrm{XhoI}$ site of the pCMV-myc vector. To allow Sindbis viral expression, GFPtagged PSD-95 (PSD-95-GFP) and GFP-tagged ADPDZ3 (GFP-PDZ3) were amplified using PCR (PSD-95-GFP: mPSD95-Mlu-S $\rightarrow$ GFP-Sph-A; GFP-ADPDZ3: GFP-Mlu$\mathrm{S} \rightarrow$ PDZ3-Sph-A) and inserted at the MluI/SphI site of the pSinRep5 vector.
The complete cDNA of mouse Kif5a (NM_008447) was amplified using PCR (mKIF5A-Bam-S $\rightarrow$ mKIF5A-Apa-A) and inserted at the BamHI/ApaI site of the pCMV-tag2B vector. To construct mutants, the KIF5A fragments were amplified using PCR and inserted at the BamHI/ApaI site of the pCMV-tag2B vector (full length: mKIF5A-Bam$\mathrm{S} \rightarrow$ mKIF5A-Apa-A; 636: mKIF5A-Bam-S $\rightarrow$ mKIF5A636-Apa-A; 826: mKIF5A-Bam-S $\rightarrow$ mKIF5A-826-Apa-A; 906: mKIF5A-Bam-S $\rightarrow$ mKIF5A-906-Apa-A; $\triangle$ MD: mKIF5A-330-Bam-S $\rightarrow$ mKIF5A-Apa-A). To allow Sindbis viral expression, wild type and $\triangle \mathrm{MD}$ mutant cDNA was amplified using PCR (mKIF5A-Sph-S $\rightarrow$ mKIF5A-Apa-A; $\triangle \mathrm{MD}: \quad$ mKIF5A-330-Sph-S $\rightarrow$ mKIF5A-Apa-A) and inserted at the SphI/ApaI site of pSinRep5-mRFP, yielding the DNA constructs encoding for mRFP-tagged WT KIF5A or mRFP-tagged $\triangle \mathrm{MD}$. The complete cDNA of mouse Kif5b (NM_008448) was amplified using PCR (mKIF5BBam-S $\rightarrow$ mKIF5B-Apa-A) and inserted at the BamHI/ ApaI site of the pCMV-tag2B vector. The complete cDNA of human Kif5c (NM_004522) was amplified using PCR (hKIF5C-R1-S $\rightarrow$ hKIF5C-Sal-A) and inserted at the EcoRI/ SalI site of the pCMV-tag2B vector. The cDNA clones of KIF5s were provided by Dr. EY Shin (Chungbuk National University). All PCR primers for PCR were purchased from Bioneer (Daejeon, Republic of Korea). Restriction enzymes used in our experiments were purchased from New England Biolabs (NEB, Ipswich, MS, USA).

mPSD95-R1-S: 5' - ggaattcaatggactgtctctgtatagtg-3', mPSD95-Xho-A: 5' -ccgctcgagtcagagtctctctcgggctg-3' PDZ1-Xho-A: 5' -ccgctcgagtcacttctcagctgggggttt-3' PDZ2-Xho-A: 5' -ccgctcgagtcaggccacctttaggtacac-3' 


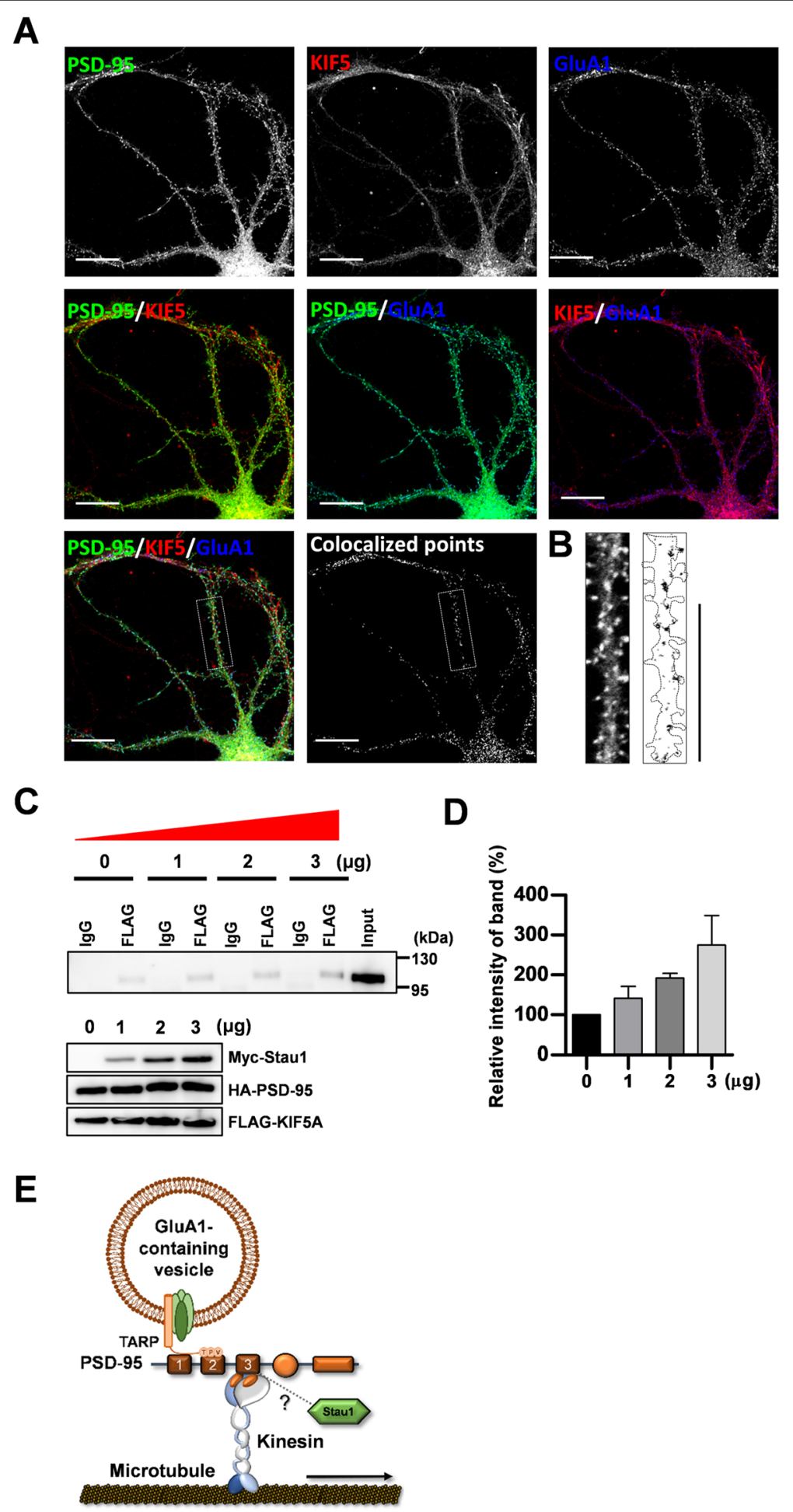

Fig. 7 (See legend on next page.) 
(See figure on previous page.)

Fig. 7 Complexes of PSD-95-KIF5 colocalized with GluA1 particles in dendrites. Cultured hippocampal neurons were transfected with PSD-95-GFP constructs and incubated for days. The cultures were immunostained with monoclonal anti-PSD-95 antibody and polyclonal GluA1 antibody; they were subsequently stained with C3-conjugated anti-mouse lgG and Alexa-Fluor 647 anti-rabbit lgG antibody. a Representative images of immunostaining in the first row. Each image was merged to show colocalization in the second row. A colocalized image of PSD-95 and KIF5 was collated with the image of GluA1. The colocalized points appeared white. Scale bar: $20 \mu \mathrm{m}$. $\mathbf{b}$ Boxed dendrites were enlarged to see the colocalization of GluA1 with the complex of PSD-95-KIF5A. c Staufen expression increased the association of PSD-95 and KIF5A. HA-PSD-95 and FLAG- KIF5A were cotransfected with 1, 2, or $3 \mu \mathrm{g}$ of Myc-Staufen, or without Myc-Staufen as a control. After immunoprecipitation using antiFLAG antibody or mouse IgG, immunoprecipitates were analyzed by Western blotting using anti-HA antibody. The lower panel shows expression of each group. d Quantified data of Western blot analyses $(0 \mu \mathrm{g}$ of Staufen: $100.0 \% \pm 0.00 \%, n=3 ; 1 \mu \mathrm{g}$ of Staufen: $141.6 \% \pm 16.96 \%, n=3 ; 2 \mu \mathrm{g}$ of Staufen: $192.3 \% \pm 6.59 \% ; 3 \mu \mathrm{g}$ of Staufen; $274.4 \% \pm 42.30 \%, n=3$ ). N values indicate the number of independent experiments. e Schematic diagram showing GluA1-containing vesicle transport mediated by PSD-95-KIF5A complex in dendrites. TARP: transmembrane AMPA receptor regulatory protein

PDZ3-Xho-A: $5^{\prime}$-ccgctcgagtcaccgcttggggttgcttcg-3 SH3-Xho-A: $5^{\prime}$-ccgctcgagtcagcgagcgtagtgcacttc-3' GMPK-R1-S: $5^{\prime}$-ggaattcacccatcatcatccttggg-3' mPSD95-ADPDZ3-R1-S: 5' -ggaattcaaagccagcaatgcctacc-3' PDZ3-Xho-A2: $5^{\prime}$-ccgctcgagtcagatgatcgtgaccgtctg-3' mPSD95-PDZ3-R1-S: 5' -ggaattcaaggcggatcgtgatccatc-3' AD-Xho-A: 5' -ccgctcgagtcaccttggttcccggggaa-3 mPSD95-Mlu-S: 5 ' -cgacgcgtatggactgtctctgtatagtg-3' GFP-Sph-A: acatgcatgcttacttgtacagctcgtcca-3' GFP-Mlu-S: 5' -cgacgcgtgtcgccaccatggtgagc-3' PDZ3-Sph-A: 5' -acatgcatgctcagatgatcgtgaccgtctg-3' mKIF5A-Bam-S: 5' -cgggatccatggcggagactaacaac-3' mKIF5A-Apa-A: 5': 5' -tgggccccttagctggctgctgtctc-3' mKIF5A-636-Apa-A: 5' -tgggggccttaatgctgtgagatgagcag-3' mKIF5A-826-Apa-A: $5^{\prime}$-tgggggccttaggaatgaatcccccac-3' mKIF5A-906-Apa-A: 5' -tgggggccttagtaccgcacggcttcttt-3' mKIF5A-330-Bam-S: 5' -cgggatccgcctcagtgaatctggag-3' mKIF5A-Sph-S: 5 ' -acatgcatgctcgaccaccatggcgga-3' mKIF5A-330-Sph-S: 5' -acatgcatgcgcctcagtgaatctggag-3' mKIF5B-Bam-S: $5^{\prime}$-cgggatccatggcggacccggcggag-3' mKIF5B-Apa-A: 5' -agggggccttacgactgcttgcctccac-3' hKIF5C-R1-S: $5^{\prime}$-ggaattctatggcggatccagccgaa-3' hKIF5C-Sal-A: 5 ' -cgacgtcgacttatttctggtagtgagtgg-3'

\section{Co-immunoprecipitation}

For co-immunoprecipitation (co-IP), cell lysates were prepared by adding lysis buffer $\left(150 \mathrm{mM} \mathrm{NaCl}, 1 \%\right.$ IGEPAL $^{\oplus}$ CA-630, $50 \mathrm{mM}$ Tris.Cl; pH 8.0) supplemented with a protease inhibitor cocktail (Roche, Basel, Switzerland). The lysate was immunoprecipitated using 2-3 $\mu \mathrm{g}$ of antibody (specificity indicated in the figures), mouse immunoglobulin G (IgG; Sigma-Aldrich, St. Louis, MO, USA), and incubated with $50 \mu \mathrm{L}$ of Protein G-Sepharose (GE Healthcare, Chicago, IL, USA). The immunoprecipitates were washed three times in $1 \mathrm{~mL}$ of ice-cold lysis buffer, followed by additional wash an additional time with $1 \mathrm{~mL}$ of $50 \mathrm{mM}$ Tris. $\mathrm{Cl}$ ( $\mathrm{pH}$ 8.0). The precipitated proteins were separated using sodium dodecyl sulfate-polyacrylamide gel electrophoresis (SDS-PAGE) (8\%-12\%). For western blot analysis, the blots were incubated using the antibody indicated in the figures. All co-IPs and western blot analyses were performed more than twice to confirm that the data were reproducible. The following antibodies were used in the co-IPs and western blot analyses: monoclonal anti-FLAG antibody (1:2000, Clone M2; Sigma-Aldrich), monoclonal anti-HA antibody (1:2000, Clone HA-7; Sigma-Aldrich), and monoclonal anti-Myc antibody (1: 2000, Clone 9E10; Sigma-Aldrich).

\section{Immunocytochemistry and proximity ligation assay}

For the immunocytochemistry, cultures were fixed using a fixative (4\% paraformaldehyde, 4\% sucrose, $\mathrm{pH} 7.2$ ) and permeabilized using PBT (0.1\% TritonX-100, $0.1 \%$ BSA in PBS). In the case of surface GluA1 immunocytochemistry, no permeabilization step was performed. The cultures were pretreated using the preblock solution ( $2 \%$ BSA, 0.08 TritonX-100 in PBS) for $1 \mathrm{~h}$ and each antibody was directly added to the preblock solution for $2 \mathrm{~h}$. The following antibodies were used for staining, each at a dilution of 1:50; monoclonal anti-PSD-95 antibody (clone 6G6-1C9; Affinity Bioreagents, Golden, CO, USA), polyclonal anti-PSD-95 antibody (Cell Signaling, Danvers, MA, USA), monoclonal anti-kinesin antibody (Clone: H2; Millipore, Temecula, CA, USA), polyclonal anti-synapsin I antibody (Millipore), polyclonal antiGluA1 antibody (Upstate, Lake Placid, NY), polyclonal anti-GluA1 antibody (Alomone Labs, Jerusalem, Israel) for surface GluA1.The following antibodies were used for secondary staining, each at a dilution of 1:200: Alexa Fluor 488 anti-rabbit IgG antibody (Molecular Probes, Eugene, OR, USA), Cy3-conjugated anti-mouse IgG antibody (Jackson ImmunoResearch Laboratories, West Grove, PA, USA), Cy3-conjugated anti-rabbit IgG antibody (Jackson ImmunoResearch Laboratories), and Alexa Fluor 647 anti-rabbit IgG antibody (Molecular Probes).

For PLA using Duolink ${ }^{\circledast}$ In Situ-Fluorescence (Sigma-Aldrich), the cultures were infected with Sindbis viruses encoding GFP to visualize whole dendritic structures and then fixed as described above; rabbit polyclonal anti-PSD-95 antibodies (Cell Signaling) and mouse monoclonal anti-KIF5 antibodies 
(Clone H2, Millipore) were then used. All procedures were performed according to the manufacturers' instructions. The nucleus of each neuron was stained with 4',6-diamidino-2-phenylindole (DAPI, Sigma-Aldrich). Immunostaining and PLA were visualized using confocal microscopy (Zeiss 710; Carl Zeiss, Oberkochen, Germany).

\section{Image analysis}

Secondary or tertiary dendrites with a similar diameter were selected from acquired neuron images and straightened using a plugin of ImageJ program (ver 1.47; National Institute of Health, Bethesda, VA, USA). The images of straightened dendrites were transited to threshold images. The number and size of PSD-95 or GluA1 particles were measured using the particle analysis plugin. Colocalization was measured from the threshold images using colocalization plugins and represented using either Pearson's correlation coefficient ( $\mathrm{R}$-value) or a percentage. All image analyses were performed by blind experiment.

\section{Statistical analysis}

Normality of the data was assessed using either the Kolmogorov-Smirnov test or the D'Agostino and Pearson omnibus normality tests. If the data followed Gaussian distribution, a Student's $t$-test was performed to determine statistical significance between two groups, while analysis of variance (ANOVA) was performed among three or more groups, with Newman Keul's analysis used as a post hoc analysis. If the data did not follow Gaussian distributions, the nonparametric Mann-Whitney test was performed to determine statistical significance between two groups, while the Kruskal-Wallis test combined with Dunn's multiple comparison test was performed among three or more groups. All statistical analyses were performed using GraphPad prism (ver 5.02; GraphPad Software, San Diego, CA, USA).

\section{Supplementary information}

Supplementary information accompanies this paper at https://doi.org/10. 1186/s13041-019-0520- $x$

Additional file 1: Figure S1. All isoforms of KIF5 interact with PSD-95. Cultured HEK cells were transfected with plasmids of HA-tagged PSD-95 and FLAG-tagged KIF5A or FLAG-tagged KIF5B, or FLAG-tagged KIF5C.

The lysates were used for IP using monoclonal anti-FLAG antibody $(2 \mu \mathrm{g}$, Clone M2, Sigma-Aldrich) and then the precipitates were analyzed by Western blotting assay using monoclonal anti-HA antibody (1:2000, Clone HA-7; Sigma-Aldrich). The bottom blots show expression of each protein used in immunoprecipitations.

Additional file 2: Figure S2. ADPDZ3 expression reduces the level of PSD-95 in dendrites. Cultured rat hippocampal neurons were infected with Sindbis viruses encoding GFP or GFP-ADPDZ3 and incubated for $9 \mathrm{~h}$ to allow expression. The cultures were then subjected to immunostaining using monoclonal anti-PSD-antibody and Cy3-conjugated anti-mouse lgG antibody. They were visualized using confocal microscopy. (A)
Representative images of expressed neurons. Arrows indicate analyzed dendrites. Scale bar: $20 \mu \mathrm{m}$. (B) ADPDZ3 expression reduced the number of PSD-95 particles per $10 \mu \mathrm{m}$ of dendrites (GFP: $5.41 \pm 0.24, n=14$, $1188 \mu \mathrm{m}$; ADPDZ3: $4.61 \pm 0.26, n=16,1542 \mu \mathrm{m})$. (C) ADPDZ3 expression did not change the size of PSD-95 particles (GFP: $0.34 \mu \mathrm{m}^{2} \pm 0.01 \mu \mathrm{m}^{2}$, $n=14,1188 \mu \mathrm{m} ;$ ADPDZ3: $\left.0.32 \mu \mathrm{m}^{2} \pm 0.01 \mu \mathrm{m}^{2}, \mathrm{n}=16,1542 \mu \mathrm{m}\right)$. (D) Representative images of dendrites divided into constant length $(25 \mu \mathrm{m})$. (D) Relative number of PSD-95 particles were reduced in the distal regions $(1-25 \mu \mathrm{m}$, GFP: $100.00 \% \pm 0.00 \%, n=13$, ADPDZ3: $100.00 \% \pm 0.00 \%, n=$ 15; 26-50 $\mu \mathrm{m}$, GFP: $92.71 \% \pm 5.93 \%, \mathrm{n}=13$, ADPDZ3: $86.32 \% \pm 6.70 \%, \mathrm{n}=$ 15; $51-75 \mu \mathrm{m}$, GFP: $76.73 \% \pm 10.34 \%, n=13$, ADPDZ3: $68.66 \% \pm 5.65 \%$, $\mathrm{n}=15 ; 76-100 \mu \mathrm{m}$, GFP: $71.60 \% \pm 7.88 \%, n=12$, ADPDZ3: $48.60 \% \pm 2.71 \%$, $\mathrm{n}=14$ ). (F) Relative size of PSD-95 particles was not changed in all dendritic regions ( $1-25 \mu \mathrm{m}$, GFP: $100.00 \% \pm 0.00 \%, \mathrm{n}=13$, ADPDZ3:

$100.00 \% \pm 0.00 \%, n=15 ; 26-50 \mu \mathrm{m}$, GFP: $98.26 \% \pm 5.83 \%, n=13$, ADPDZ3: $111.98 \% \pm 7.62 \%, n=15 ; 51-75 \mu \mathrm{m}$, GFP: $100.87 \% \pm 7.50 \%, n=13$, ADPDZ3: $108.83 \% \pm 6.05 \%, n=15 ; 76-100 \mu m$, GFP: $109.74 \% \pm 9.18 \%, n=$ 12, ADPDZ3: $120.50 \% \pm 11.38 \%, n=14)$. $N$ values indicate $n$ dendrites from $\mathrm{n}$ neurons.

\section{Abbreviations}

AD: Association domain; AMPA: a-Amino-3-hydroxy-5-methyl-4isoxazolepropionic acid; ANOVA: Analysis of variance; CASK: Calcium/ calmodulin-dependent serine protein kinase; DAPI: 4',6-Diamidino-2phenylindole; DLG: Discs large homology; GABA: Y-Aminobutyric acid; GFP: Green fluorescent protein; GluA1: Glutamate receptor 1; GRIP1: Glutamate receptor interacting protein 1; HA: Hemagglutinin; HEK: Human embryonic kidney; IgG: Immunoglobulin G; KIF: Kinesin superfamily protein; MAGI-2: Membrane associated guanylate kinase inverted-2; MAGUK: Member of membrane-associated guanylate kinase; NMDA: N-methyl-D-aspartate; PBS: Phosphate buffered saline; PLA: Proximity ligation assay; PSD-95: Postsynaptic density protein 95; SAP97: Synapseassociated protein-97; S-SCAM: Synaptic scaffolding molecule;

TARP: Transmembrane AMPA receptor regulatory protein; TrkB: Tyrosine receptor kinase B; ZO-1: Zona occludens-1

\section{Acknowledgements}

We are very grateful to EY Shin for sharing CDNA clones of mouse KIF5A, $\mathrm{KIF} 5 \mathrm{~B}$, and human KIF5C

\section{Authors' contributions}

KSY, JYO, and HL performed the cell biological and biochemical experiments. $\mathrm{KL}$ performed the Staufen association experiment. HKK designed the experiments. HP, KSY, YSP and HKK analyzed and interpreted the results. HP and HKK wrote the manuscript. All authors reviewed and approved the manuscript.

\section{Funding}

This research was supported by Basic Science Research Program through the National Research Foundation of Korea (NRF) funded by the Ministry of Education (NRF-2015R1D1A1A01059654).

Availability of data and materials

All data generated or analyzed during this study are included in this published article and its supplementary information files.

\section{Ethics approval and consent to participate}

Rat hippocampal neuron cultures were performed in accordance with the approved animal protocols and the guidelines of the Institutional Animal Care and Use Committee of Chungbuk National University (CBNUA-1049-1701).

\section{Competing interests}

The authors declare that they have no competing interests.

\section{Author details}

'Department of Medicine and Microbiology, Graduate Program in Neuroscience, College of Medicine, Chungbuk National University, 1 Chungdae-ro, Seowon-gu, Cheongju 28644, South Korea. ${ }^{2}$ Department of Structure and Function of Neural Network, Korea Brain Research Institute, 61 
Cheomdan-ro, Dong-gu, Daegu 41068, South Korea. ${ }^{3}$ Department of Neurosurgery, Graduate Program in Neuroscience, College of Medicine, Chungbuk National University, 1 Chungdae-ro, Seowon-gu, Cheongju 28644, South Korea.

\section{Received: 14 February 2019 Accepted: 4 November 2019}

Published online: 21 November 2019

\section{References}

1. Ehrlich I, Malinow R. Postsynaptic density 95 controls AMPA receptor incorporation during long-term potentiation and experience-driven synaptic plasticity. J Neurosci. 2004;24(4):916-27.

2. El-Husseini AE, Schnell E, Chetkovich DM, Nicoll RA, Bredt DS. PSD-95 involvement in maturation of excitatory synapses. Science. 2000;290(5495): 1364-8.

3. El-Husseini Ael D, Schnell E, Dakoji S, Sweeney N, Zhou Q, Prange O, Gauthier-Campbell C, Aguilera-Moreno A, Nicoll RA, Bredt DS. Synaptic strength regulated by palmitate cycling on PSD-95. Cell. 2002;108(6):849-63.

4. Huganir RL, Nicoll RA. AMPARs and synaptic plasticity: the last 25 years. Neuron. 2013;80(3):704-17.

5. Diering $\mathrm{GH}$, Huganir RL. The AMPA receptor code of synaptic plasticity. Neuron. 2018:100(2):314-29.

6. $\quad$ Feng $W$, Zhang M. Organization and dynamics of PDZ-domain-related supramodules in the postsynaptic density. Nat Rev Neurosci. 2009;10(2):87-99.

7. Kim E, Sheng M. PDZ domain proteins of synapses. Nat Rev Neurosci. 2004; 5(10):771-81.

8. Liang $H$, Wang $H$, Wang $S$, Francis $R$, Paxinos $G$, Huang X. 3D imaging of PSD-95 in the mouse brain using the advanced CUBIC method. Mol Brain. 2018;11(1):50

9. Schnell E, Sizemore M, Karimzadegan S, Chen L, Bredt DS, Nicoll RA. Direct interactions between PSD-95 and stargazin control synaptic AMPA receptor number. Proc Natl Acad Sci U S A. 2002;99(21):13902-7.

10. Elias GM, Funke L, Stein V, Grant SG, Bredt DS, Nicoll RA. Synapse-specific and developmentally regulated targeting of AMPA receptors by a family of MAGUK scaffolding proteins. Neuron. 2006;52(2):307-20.

11. Zhang P, Lisman JE. Activity-dependent regulation of synaptic strength by PSD-95 in CA1 neurons. J Neurophysiol. 2012;107(4):1058-66.

12. Zheng CY, Seabold GK, Horak M, Petralia RS. MAGUKs, synaptic development, and synaptic plasticity. Neuroscientist. 2011;17(5):493-512.

13. Grant SG. Synaptopathies: diseases of the synaptome. Curr Opin Neurobiol. 2012;22(3):522-9.

14. de Bartolomeis A, Latte G, Tomasetti C, lasevoli F. Glutamatergic postsynaptic density protein dysfunctions in synaptic plasticity and dendritic spines morphology: relevance to schizophrenia and other behavioral disorders pathophysiology, and implications for novel therapeutic approaches. Mol Neurobiol. 2014;49(1):484-511.

15. Mok H, Shin H, Kim S, Lee JR, Yoon J, Kim E. Association of the kinesin superfamily motor protein KIF1Balpha with postsynaptic density-95 (PSD95), synapse-associated protein-97, and synaptic scaffolding molecule PSD95/discs large/zona occludens-1 proteins. J Neurosci. 2002;22(13):5253-8.

16. Hirokawa N, Takemura R. Molecular motors and mechanisms of directional transport in neurons. Nat Rev Neurosci. 2005;6(3):201-14.

17. Kanai Y, Okada Y, Tanaka Y, Harada A, Terada S, Hirokawa N. KIF5C, a novel neuronal kinesin enriched in motor neurons. J Neurosci. 2000; 20(17):6374-84.

18. Hirokawa N, Niwa S, Tanaka Y. Molecular motors in neurons: transport mechanisms and roles in brain function, development, and disease. Neuron. 2010;68(4):610-38.

19. Nakajima K, Yin X, Takei Y, Seog DH, Homma N, Hirokawa N. Molecular motor KIF5A is essential for GABA(a) receptor transport, and KIF5A deletion causes epilepsy. Neuron. 2012;76(5):945-61.

20. Terada S, Kinjo M, Aihara M, Takei Y, Hirokawa N. Kinesin-1/Hsc70dependent mechanism of slow axonal transport and its relation to fast axonal transport. EMBO J. 2010;29(4):843-54.

21. Setou M, Nakagawa T, Seog DH, Hirokawa N. Kinesin superfamily motor protein KIF17 and mLin-10 in NMDA receptor-containing vesicle transport. Science. 2000;288(5472):1796-802.

22. Heisler FF, Lee HK, Gromova KV, Pechmann Y, Schurek B, Ruschkies L, Schroeder M, Schweizer M, Kneussel M. GRIP1 interlinks N-cadherin and AMPA receptors at vesicles to promote combined cargo transport into dendrites. Proc Natl Acad Sci U S A. 2014;111(13):5030-5.
23. Asaba N, Hanada T, Takeuchi A, Chishti AH. Direct interaction with a kinesin-related motor mediates transport of mammalian discs large tumor suppressor homologue in epithelial cells. J Biol Chem. 2003; 278(10):8395-400.

24. Hanada T, Lin L, Tibaldi EV, Reinherz EL, Chishti AH. GAKIN, a novel kinesin-like protein associates with the human homologue of the Drosophila discs large tumor suppressor in T lymphocytes. J Biol Chem. 2000;275(37):28774-84.

25. Kanai Y, Dohmae N, Hirokawa N. Kinesin transports RNA: isolation and characterization of an RNA-transporting granule. Neuron. 2004;43(4):513-25.

26. Oh JY, Kwon A, Jo A, Kim H, Goo YS, Lee JA, Kim HK. Activity-dependent synaptic localization of processing bodies and their role in dendritic structural plasticity. J Cell Sci. 2013;126(Pt 9):2114-23.

27. Seiler S, Kirchner J, Horn C, Kallipolitou A, Woehlke G, Schliwa M. Cargo binding and regulatory sites in the tail of fungal conventional kinesin. Nat Cell Biol. 2000;2(6):333-8.

28. Setou M, Seog DH, Tanaka Y, Kanai Y, Takei Y, Kawagishi M, Hirokawa N. Glutamate-receptor-interacting protein GRIP1 directly steers kinesin to dendrites. Nature. 2002;417(6884):83-7.

29. Opazo P, Sainlos M, Choquet D. Regulation of AMPA receptor surface diffusion by PSD-95 slots. Curr Opin Neurobiol. 2012;22(3):453-60.

30. Jeong JH, Nam YJ, Kim SY, Kim EG, Jeong J, Kim HK. The transport of Staufen2-containing ribonucleoprotein complexes involves kinesin motor protein and is modulated by mitogen-activated protein kinase pathway. J Neurochem. 2007;102(6):2073-84

31. Oh JY, Lim CS, Yoo KS, Park H, Park YS, Kim EG, Lee YS, Kaang BK, Kim HK Adenomatous polyposis coli-stimulated GEF 1 (Asef1) is a negative regulator of excitatory synaptic function. J Neurochem. 2018;147(5):595-608.

32. Lin Y, Jones BW, Liu A, Vazquez-Chona FR, Lauritzen JS, Ferrell WD, Marc RE. Rapid glutamate receptor 2 trafficking during retinal degeneration. Mol Neurodegener. 2012;7:7.

33. Miki H, Setou M, Kaneshiro K, Hirokawa N. All kinesin superfamily protein, KIF, genes in mouse and human. Proc Natl Acad Sci U S A 2001;98(13):7004-11.

34. Conforti L, Dell'Agnello C, Calvaresi N, Tortarolo M, Giorgini A, Coleman MP, Bendotti C. Kif1Bbeta isoform is enriched in motor neurons but does not change in a mouse model of amyotrophic lateral sclerosis. J Neurosci Res. 2003;71(5):732-9.

35. Aizawa H, Sekine Y, Takemura R, Zhang Z, Nangaku M, Hirokawa N. Kinesin family in murine central nervous system. J Cell Biol. 1992;119(5):1287-96.

36. Jeyifous O, Waites CL, Specht CG, Fujisawa S, Schubert M, Lin El, Marshall J, Aoki C, de Silva T, Montgomery JM, et al. SAP97 and CASK mediate sorting of NMDA receptors through a previously unknown secretory pathway. Nat Neurosci. 2009:12(8):1011-9.

37. Twelvetrees AE, Yuen EY, Arancibia-Carcamo IL, MacAskill AF, Rostaing P, Lumb MJ, Humbert S, Triller A, Saudou F, Yan Z, et al. Delivery of GABAARs to synapses is mediated by HAP1-KIF5 and disrupted by mutant huntingtin. Neuron. 2010:65(1):53-65.

38. Verhey KJ, Rapoport TA. Kinesin carries the signal. Trends Biochem Sci. 2001; 26(9):545-50.

39. Nourry C, Grant SG, Borg JP. PDZ domain proteins: plug and play! Sci STKE. 2003;2003(179):RE7.

\section{Publisher's Note}

Springer Nature remains neutral with regard to jurisdictional claims in published maps and institutional affiliations.

Ready to submit your research? Choose BMC and benefit from:

- fast, convenient online submission

- thorough peer review by experienced researchers in your field

- rapid publication on acceptance

- support for research data, including large and complex data types

- gold Open Access which fosters wider collaboration and increased citations

- maximum visibility for your research: over $100 \mathrm{M}$ website views per year

At $\mathrm{BMC}$, research is always in progress.

Learn more biomedcentral.com/submission 